\title{
Management of severe intraoperative hemorrhage during intraventricular neuroendoscopic procedures: the dry field technique
}

\author{
Joachim Oertel, MD, ${ }^{1}$ Stefan Linsler, MD, ${ }^{1}$ Akos Csokonay, MD, ${ }^{1}$ Henry W. S. Schroeder, MD, PhD, ${ }^{2}$ \\ and Sebastian Senger, MD'
}

1Department of Neurosurgery, Medical School of the Saarland University, Homburg (Saar); and 'Department of Neurosurgery, Ernst Moritz Arndt University, Greifswald, Germany

\begin{abstract}
OBJECTIVE The unexpected intraoperative intraventricular hemorrhage is a rare but feared and life-threatening complication in neuroendoscopic procedures because of loss of endoscopic vision. The authors present their experience with the so-called "dry field technique" (DFT) for the management of intraventricular hemorrhages during purely endoscopic procedures. This technique requires the aspiration of the entire intraventricular CSF to achieve clear visualization of the bleeding source.
\end{abstract}

METHODS More than 500 neuroendoscopic intraventricular procedures were retrospectively analyzed over the last 24 years for documented severe hemorrhages, which were treated by the application of the DFT.

RESULTS The technique was required in 6 cases, including tumor resection/biopsy, cyst resection, and intraventricular lavage. Additionally, the technique was applied as part of the planned strategy in 3 cases of endoscopic tumor removal. The hemorrhage was stopped in all cases and no associated postoperative deficits occurred.

CONCLUSIONS Although severe hemorrhages are rare, the neurosurgeon needs to be aware of them and has to establish strategies for their management. Most hemorrhages can be stopped by constant irrigation and coagulation. In the other rare cases, the DFT is a safe, reliable technique and can be easily incorporated into endoscopic surgery.

https://thejns.org/doi/abs/10.3171/2018.4.JNS172537

KEYWORDS neuroendoscopy; dry field technique; intraventricular hemorrhage; complication management; surgical technique

$\mathrm{N}$ EUROSURGEONS worldwide have been gaining experience with intraventricular endoscopy over the last several decades. Procedures such as endoscopic ventriculostomy have become standardized neuroendoscopic procedures in many departments. ${ }^{8,15}$ In addition, more sophisticated procedures such as tumor or cyst resection have become more readily available in the neurosurgical community with comparably low complication rates. ${ }^{2}$ Reports of intraoperative hemorrhages during intraventricular neuroendoscopic procedures are approximately $3 \%{ }^{4,9,10,16}$ A recent meta-analysis evaluated 41 hemorrhages in 592 cases. ${ }^{1}$ A multicenter study on neuroendoscopic biopsies revealed "mild" bleeding in 221 of 293 patients. ${ }^{6}$ As Li et al. have reported in their recent study, most of those bleeding events were minor bleedings without vision impairment and with short bleeding time after irrigation. ${ }^{11}$ There is an ongoing debate about the definition of hemorrhage complication, as short bleed- ings-which may occur after taking a biopsy specimen of pathological tissue-appear to be unavoidable and might not be counted as a complication. Some authors only count those bleeding events as complications that lead to a discontinuation of the surgery or a switch to microsurgery. ${ }^{6}$

Despite this lack of a consensus regarding the definition of hemorrhage complication, a severe intraoperative hemorrhage bears a life-threatening risk. Therefore, the authors wanted to share their experience with management of intraoperative bleeding in severe cases during fully endoscopic surgery. The so-called "dry field technique" (DFT) was first described during the resection of a colloid cyst and two intraventricular tumors. ${ }^{16,17}$

\section{Methods \\ Data Collection}

This report includes records of neuroendoscopic in- 
TABLE 1. Summary of all patients with unexpected severe intraventricular hemorrhage during intraventricular neuroendoscopic procedures

\begin{tabular}{|c|c|c|c|c|c|c|}
\hline $\begin{array}{l}\text { Case } \\
\text { No. }\end{array}$ & $\begin{array}{l}\text { Age (yrs), } \\
\text { Sex }\end{array}$ & Pathology & Initial Symptoms & Surgical Procedure & Outcome & $\begin{array}{c}\text { Add'l } \\
\text { Procedures }\end{array}$ \\
\hline 1 & $62, \mathrm{~F}$ & Hemangioma of side ventricle & Organic mental disorder & Tumor resection & Symptom free & None \\
\hline 2 & $60, \mathrm{~F}$ & Intraventricular hemorrhage & Comatose & Clot removal, ventricular lavage & Unchanged & EVD \\
\hline 3 & $61, \mathrm{M}$ & Colloid cyst & Headache, memory disturbance & Cyst resection, septostomy & Symptom free & None \\
\hline 4 & $48, M$ & $\begin{array}{l}\text { Plexus papilloma of 3rd } \\
\text { ventricle }\end{array}$ & Seizure & Tumor resection & Symptom free & None \\
\hline 5 & $48, F$ & Glioblastoma of thalamus & $\begin{array}{l}\text { Hydrocephalus, memory } \\
\text { disturbance }\end{array}$ & Tumor biopsy, ETV & Improved & $\begin{array}{l}\text { VP shunt after } \\
1 \mathrm{mo}\end{array}$ \\
\hline 6 & $56, \mathrm{M}$ & Colloid cyst & Headache, depression & Cyst resection & Improved & EVD \\
\hline
\end{tabular}

Add'l = additional; ETV = endoscopic third ventriculostomy; EVD = external ventricular drain; VP = ventriculoperitoneal.

traventricular procedures from the Department of Neurosurgery, Ernst Moritz Arndt University, in Greifswald, Germany, between January 1993 and March 2003; from the Department of Neurosurgery, Hannover Nordstadt Hospital, in Hanover, Germany, between April 2003 and September 2008; from the Department of Neurosurgery, Johannes Gutenberg University, in Mainz, Germany, between October 2008 and November 2010; and finally, from the Department of Neurosurgery, Saarland Medical School, in Homburg, Germany, between December 2010 and March 2018.

Records were examined retrospectively for severe bleeding events in which the DFT was applied. Those procedures included third ventriculostomy, tumor biopsy/ resection, cyst fenestration/resection, aqueductoplasty, aqueductal stenting, septostomy, hematoma clot removal, and catheter removal/placement. The procedures were mainly performed with the GAAB I and later with the GAAB II neuroendoscopy set (Karl Storz Co.).

In the experience of the authors, neuroendoscopists have to be aware of potential hemorrhage during biopsy or fenestration procedures. The risk might be lower when the specific region is coagulated beforehand. Nonetheless, a small amount of hemorrhage may occur during the tissue penetration. This may not cause impaired vision and can be flushed out by short irrigation. The authors recommend lactated Ringer's solution at $36^{\circ}-37^{\circ} \mathrm{C}$ over saline to reduce the risk of postoperative feverlike symptoms. The surgeon has to provide a sufficient outflow of the irrigation fluid to avoid life-threatening intracranial pressure (ICP) increase. Prolonged hemorrhage caused by smaller blood vessels can be stopped by patient irrigation and optional coagulation. The potential thermal damage to adjacent tissue has to be considered.

\section{The DFT}

However, if the hemorrhage cannot be controlled by these methods, the DFT should be considered. The steps of the DFT are as follows. First, a transparent sucking device-for example, a pediatric suction device provided by the anesthesiologists-is introduced in the working channel and the complete CSF is carefully aspirated. In the next step, the trocar is carefully placed over the presump- tive bleeding source. The hemorrhage should now stop after a certain amount of time. Gravity and the air environment (surgical field no longer under CSF/water) supports the clot formation. Vision is no longer impaired due to the missing CSF environment and identification of the injured vessel should be achieved. Coagulation is performed. After the hemorrhage is under control, the remaining blood clots at the bottoms of the ventricles can be aspirated under direct view. The initial procedure can be resumed and finished under the air environment or the ventricles can be carefully refilled with Ringer's solution. The final inspection should guarantee free-floating CSF without any obstructions at the aqueduct or newly formed stoma. An additional external ventricular drainage might be helpful, if postoperative increased ICP is expected or additional clearance of the CSF is necessary.

Although the indication for this technique is rare, the authors retrospectively analyzed their database to share their experience using this technique with the neurosurgical community.

\section{Results}

The analysis of more than 500 neuroendoscopic intraventricular procedures revealed only 6 cases with extensive hemorrhage that could not be managed by constant irrigation and coagulation, hence the DFT was applied. The average duration of irrigation was approximately 12 minutes, with up to 34 minutes in the case of a colloid cyst. Pathologies and procedures are summarized in Table 1. Tumor resection or biopsy was scheduled in 3 cases and achieved after the unexpected severe hemorrhage was controlled by the DFT. The patient suffering from a glioblastoma required an additional shunt procedure after 1 month due to the blockade of the foramen of Monro and died in the following months. The patient with an intraventricular hemorrhage remained comatose. The 2 patients suffering from a colloid cyst improved and showed no signs of cyst recurrence in the follow-up. No hemorrhage-associated transient or permanent deficits occurred. During the application of the DFT no collapse of the ventricles was noted. No complications associated with postoperative pneumocephalus were observed. An example of the DFT and postoperative CT scans is demonstrated in Fig. 1. 

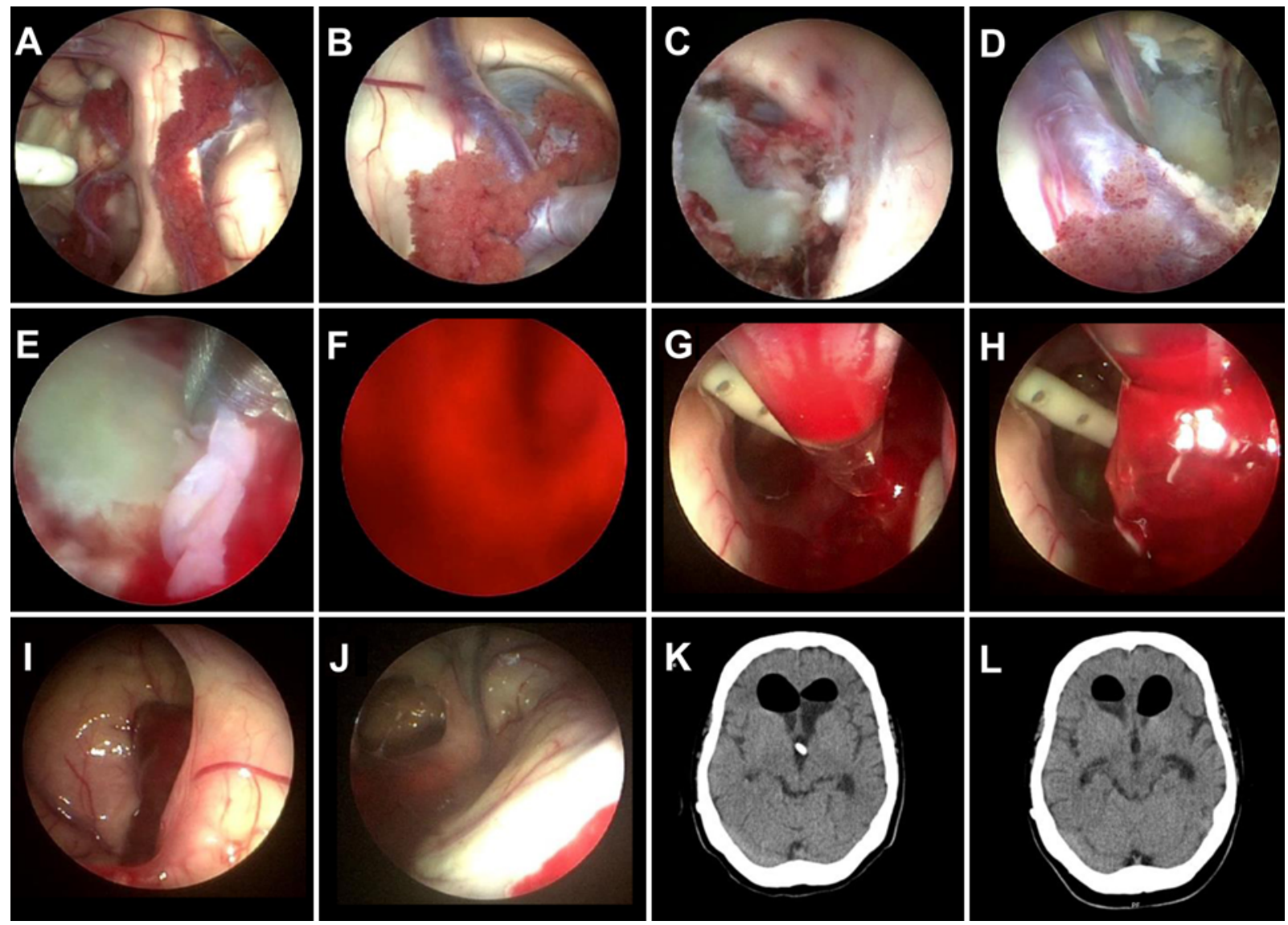

FIG. 1. Case 6. Example of intraoperative hemorrhage management. The patient, pretreated with a shunt, was scheduled for an endoscopic resection of a colloid cyst (A and B). After cyst fenestration, the mucoid part was aspirated ( $\mathbf{C}$ and $\mathbf{D})$. After removal of the cyst walls, an intraoperative hemorrhage occurred during the last steps of cyst wall resection (E and $F)$. As the hemorrhage was persistent under constant irrigation after more than 30 minutes, the CSF was completely aspirated and hemostasis was achieved using the DFT (G-I). Final inspection and postoperative CT scans obtained 24 and 72 hours after surgery (J-L) showing complete hemostasis. Figure is available in color online only.

The DFT was also applied in 3 cases of tumor resection as part of the preoperative strategy. As a high vascularization was expected, the DFT was applied to support intraoperative hemostasis. In one case of astrocytoma at the wall of the frontal horn, the patient was positioned to have the tumor at the highest point. CSF was partially aspirated, so the biopsy could be performed under clear vision (Fig. 2). Another patient suffered from a subependymoma, and in this case the CSF was aspirated to achieve complete tumor resection using an ultrasound aspirator (Soering Co.) under clear vision. In both cases the postoperative course was uneventful (Fig. 3). A third patient suffered from hydrocephalic symptoms. The MRI showed a tumor at the left basal ganglia occluding the foramen of Monro. The exophytic part of the tumor was resected using the DFT due to high vascularization (Video 1).

VIDEO 1. Clip showing 2 cases undergoing DFT. The first case (Case 6) refers to severe hemorrhage after the removal of a colloid cyst (Fig. 1). The second case (third elective case) demonstrates the DFT during the partial resection of a meningioma of the left-side ventricle. Copyright Sebastian Senger. Published with permission. Click here to view.

The histopathological analysis revealed a meningioma.
The patient recovered and showed only partial facial nerve palsy after 3 months' follow-up.

\section{Discussion}

There are several reports about the management of intraoperative hemorrhage during neuroendoscopic procedures. It is common sense that there is a stepwise escalation of the bleeding management: first irrigation, then cauterization, and lastly, more invasive methods are recommended. The most common recommendation is the conversion to a microsurgical approach..$^{5,711}$ This conversion is most commonly performed by extending the craniotomy, opening the dura, and following the trajectory of the endoscopic approach with the help of retractors. The aspiration of the intraventricular CSF is also necessary. This surgical technique might be the easiest to be adopted. However, some limitations have to be considered. Visualization of the surgical field through the microscope might be limited and the origin of the hemorrhage may not be visualized. Therefore, more invasive retraction might be necessary and causes additional damage to the brain tissue. The minimally invasive as- 

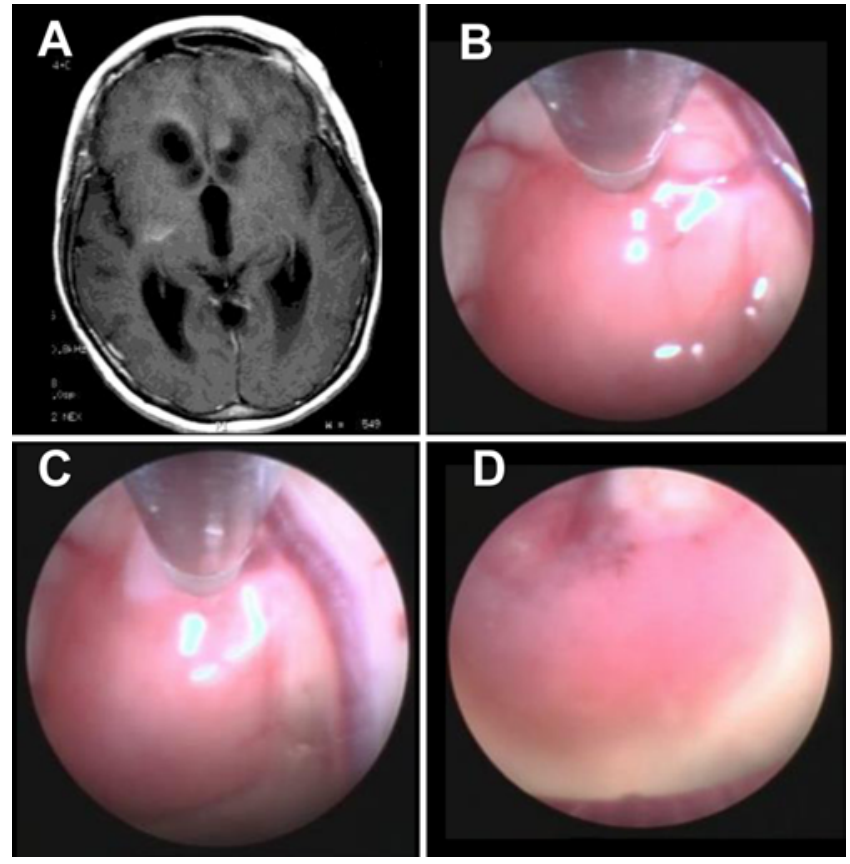

FIG. 2. Use of the DFT during the endoscopic resection of a grade III astrocytoma. A: Preoperative MR image shows the tumor at the frontal horn. B-D: Intraoperative views using the GAAB II neuroendoscopy set. Note the superficial vein on the tumor surface, requiring coagulation. Figure is available in color online only.

pect of the endoscopic approach is abandoned with this technique.

Additional strategies have been developed. Cappabianca et al. applied a small cotton pad through the working sheet and forced it into a position as a tamponade under endoscopic view. After a short period of pressure, hemostasis should be achieved. ${ }^{3}$

Special tools were developed to manage the hemorrhage endoscopically. Nagasaka et al. introduced a multifunctional suction cannula (Fujita Medical Instruments) and the "balanced irrigation, suction, coagulation technique." 13 Nishihara et al. developed a transparent working sheath for the evacuation of intraventricular and intracerebral hematoma. ${ }^{14}$ Such a working sheath is considered helpful when the bleeding source originates along the approach and is usually covered by the working sheath.

Another minimally invasive technique for hemorrhage management is the so-called "small-chamber irrigation technique."'12 In short, the endoscope is withdrawn 0.5-1 $\mathrm{cm}$ into the endoscope sheath under constant irrigation and placed over the bleeding zone. This creates a small chamber in which the hemorrhage can be stopped by irrigation or coagulation. Of course, the surgeon has to resist the temptation to withdraw the endoscope and has to be confident with the intraventricular navigation, even under impaired vision.

The presented DFT may raise some concerns about potential side effects. A collapse of the ventricle systems has thus far not been observed. This is a limited cases series of only 9 patients, although a ventricle collapse is a rather rare observation in neuroendoscopic procedures. ${ }^{7}$ Further-
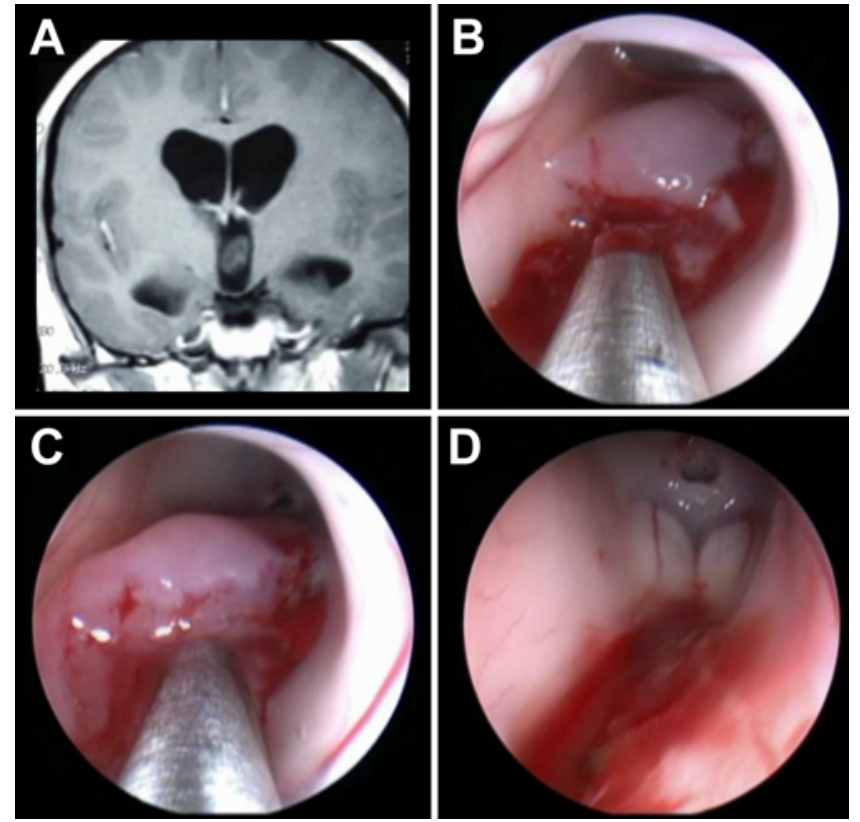

FIG. 3. Use of the DFT during endoscopic resection of a subependymoma. A: Preoperative MR image shows the tumor at the third ventricle. $B$ and C: Intraoperative views using the GAAB II neuroendoscopy set. Resection of the tumor was performed with application of an ultrasound aspirator during DFT. Note the excellent vision despite the small blood clot at the floor. D: Overview with complete hemostasis after complete resection and additional endoscopic third ventriculostomy. Figure is available in color online only.

more, potential postoperative subdural hematoma was not observed in our case series. Feverlike symptoms are not unusual after intraventricular endoscopic procedures and might occur more often after extended irrigation. However, these symptoms show spontaneous relief after a few days. A similar report about the application of the DFT in a case series of 5 pediatric and 2 adult patients did not find any technique-related complications. ${ }^{18}$

\section{Conclusions}

Severe hemorrhages are rather rare complications, and every neurosurgeon has developed his or her own strategies to manage them. That is why we want to share our experience with the neurosurgical community and offer alternative, purely endoscopic strategies to the more invasive conversion to microsurgical approaches. We believe that the DFT is a quick and safe method for bleeding management, even for less experienced neuroendoscopists. Overall, it does not require changing the instruments, and provides a broad overview of the anatomical structures and thereby orientation within the ventricular system. It can also be considered for endoscopic tumor resection from the beginning, when the tumor appears to be highly vascularized and bleedings are strongly suspected.

\section{References}

1. Barber SM, Rangel-Castilla L, Baskin D: Neuroendoscopic 
resection of intraventricular tumors: a systematic outcomes analysis. Minim Invasive Surg 2013:898753, 2013

2. Beems T, Grotenhuis JA: Long-term complications and definition of failure of neuroendoscopic procedures. Childs Nerv Syst 20:868-877, 2004

3. Cappabianca P, Cinalli G, Gangemi M, Brunori A, Cavallo LM, de Divitiis E, et al: Application of neuroendoscopy to intraventricular lesions. Neurosurgery 62 (Suppl 2):575598, 2008

4. Cinalli G, Spennato P, Ruggiero C, Aliberti F, Trischitta V, Buonocore MC, et al: Complications following endoscopic intracranial procedures in children. Childs Nerv Syst 23:633-644, 2007

5. Chowdhry SA, Cohen AR: Intraventricular neuroendoscopy: complication avoidance and management. World Neurosurg 79 (2 Suppl):15.e1-15.e10, 2013

6. Constantini S, Mohanty A, Zymberg S, Cavalheiro S, Mallucci C, Hellwig D, et al: Safety and diagnostic accuracy of neuroendoscopic biopsies: an international multicenter study. J Neurosurg Pediatr 11:704-709, 2013

7. da C F Pinto PH, Nigri F, Gobbi GN, Caparelli-Daquer EM: Conversion technique from neuroendoscopy to microsurgery in ventricular tumors: Technical note. Surg Neurol Int 7 (Suppl 31):S785-S789, 2016

8. Di Vincenzo J, Keiner D, Gaab MR, Schroeder HW, Oertel JM: Endoscopic third ventriculostomy: preoperative considerations and intraoperative strategy based on 300 procedures. J Neurol Surg A Cent Eur Neurosurg 75:20-30, 2014

9. Giannetti AV, Alvarenga AY, de Lima TO, Pedrosa HA, Souweidane MM: Neuroendoscopic biopsy of brain lesions: accuracy and complications. J Neurosurg 122:34-39, 2015

10. Hopf NJ, Grunert P, Fries G, Resch KD, Perneczky A: Endoscopic third ventriculostomy: outcome analysis of 100 consecutive procedures. Neurosurgery 44:795-806, 1999

11. Li C, Zong X, Wang X, Gui S, Zhang Y: Intraoperative hemorrhage in ventriculoscopic surgery: experience of a single Chinese neurosurgery center. World Neurosurg 88:548551, 2016

12. Manwaring JC, El Damaty A, Baldauf J, Schroeder HW: The small-chamber irrigation technique (SCIT): a simple maneuver for managing intraoperative hemorrhage during endoscopic intraventricular surgery. Neurosurgery 10 (Suppl 3):375-379, 2014

13. Nagasaka T, Tsugeno M, Ikeda H, Okamoto T, Takagawa Y, Inao S, et al: Balanced irrigation-suction technique with a multifunctional suction cannula and its application for intraoperative hemorrhage in endoscopic evacuation of intracerebral hematomas: technical note. Neurosurgery 65:E826E827, 2009
14. Nishihara T, Teraoka A, Morita A, Ueki K, Takai K, Kirino $\mathrm{T}$ : A transparent sheath for endoscopic surgery and its application in surgical evacuation of spontaneous intracerebral hematomas. Technical note. J Neurosurg 92:1053-1055, 2000

15. Oertel JM, Baldauf J, Schroeder HW, Gaab MR: Endoscopic options in children: experience with 134 procedures. J Neurosurg Pediatr 3:81-89, 2209, 2009

16. Schroeder HW, Gaab MR: Endoscopic resection of colloid cysts. Neurosurgery 51:1441-1445, 2002

17. Schroeder HW, Oertel J, Gaab MR: Incidence of complications in neuroendoscopic surgery. Childs Nerv Syst 20:878-883, 2004

18. Turhan T: Dry-field maneuver for controlling the massive intraventricular bleeding during neuroendoscopic procedures. Childs Nerv Syst 34:541-545, 2018

\section{Disclosures}

Joachim Oertel and Henry Schroeder are consultants for Karl Storz Company.

\section{Author Contributions}

Conception and design: Oertel, Schroeder, Senger. Acquisition of data: Oertel, Csokonay, Schroeder, Senger. Analysis and interpretation of data: Oertel, Senger. Drafting the article: Oertel, Linsler, Csokonay, Senger. Critically revising the article: Oertel, Linsler, Schroeder, Senger. Reviewed submitted version of manuscript: Oertel, Linsler, Schroeder, Senger. Approved the final version of the manuscript on behalf of all authors: Oertel. Study supervision: Oertel.

\section{Supplemental Information Videos}

Video 1. https://vimeo.com/270103358.

\section{Correspondence}

Joachim Oertel: Universität des Saarlandes, Homburg, Germany. joachim.oertel@uks.eu. 